\title{
Medical Image of the Week: Staphylococcal Pneumonia in a Patient with Influenza
}

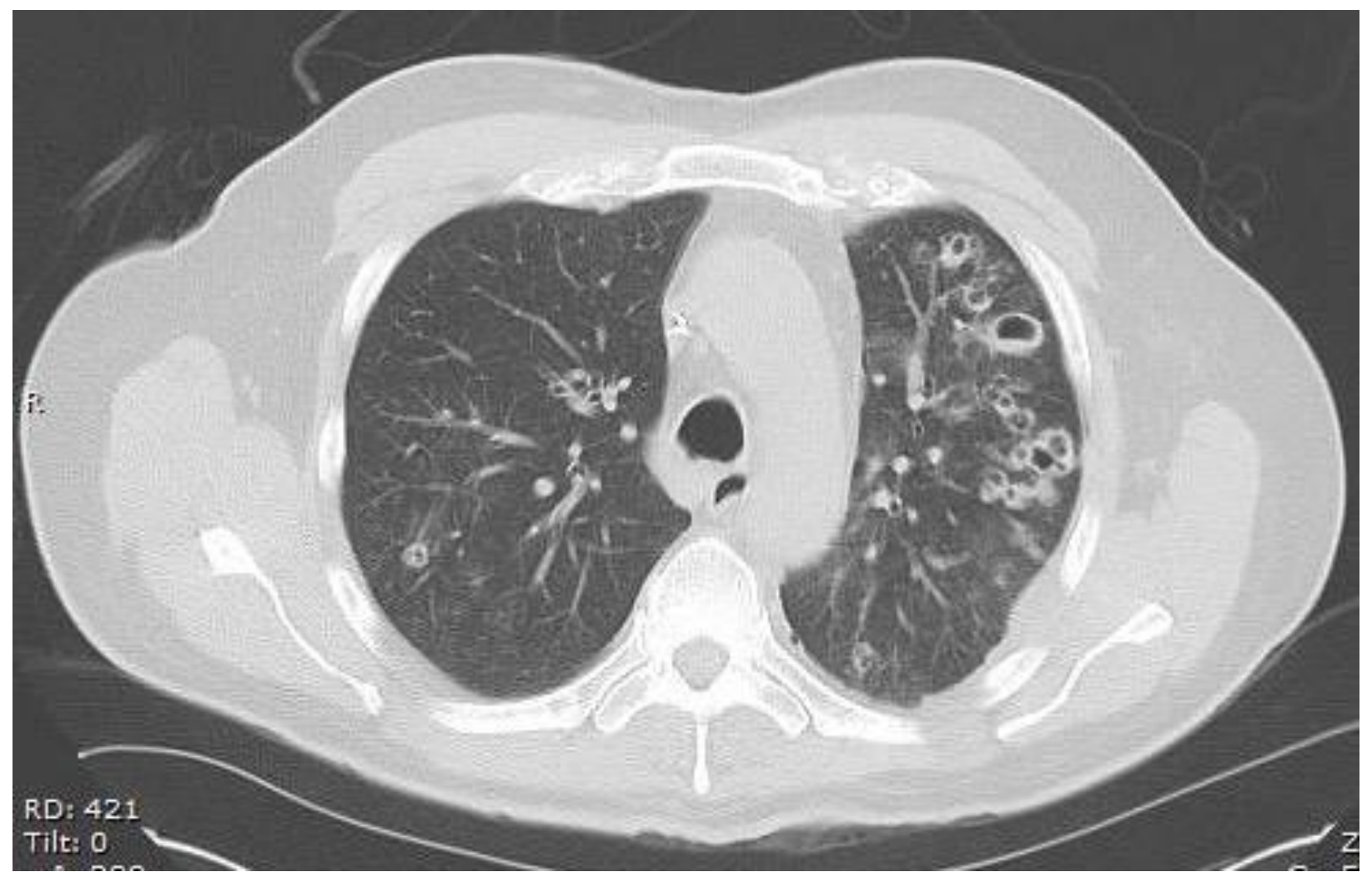

Figure 1. Thoracic CT scan axial view demonstrating bilateral cavitary infiltrates.

A 63-year-old, obese diabetic man presented to his primary care physician with complaints of fever, headache, myalgias, and cough. A nasal swab specimen was positive for influenza A by fluorescent immunoassay. Therapy with oseltamivir was initiated. The patient's symptoms progressed and he was transported to the emergency department, where he was found to have a room air oxygen saturation of $74 \%$, bilateral basilar infiltrates on chest radiograph, a white blood count of $24.2 \mathrm{~K} / \mathrm{uL}$ and a procalcitonin level of $12.66 \mathrm{ng} / \mathrm{ml}$. He was placed on BIPAP with high flow supplemental oxygen, started on empiric intravenous antibiotic therapy with vancomycin and piperacillin/tazobactam, and admitted to the intensive care unit. Blood and sputum cultures were eventually positive for methicillin-sensitive Staphylococcus aureus, and the patient's antibiotic therapy was de-escalated to nafcillin. On hospital day 5, a CT of the chest obtained to evaluate pleuritic pain revealed extensive bilateral cavitary infiltrates (Figure 1). The patient's discomfort resolved without further intervention, he continued to improve, and was uneventfully transitioned to oral therapy.

S. aureus pneumonia is characterized by high fever, productive cough, and a radiographic pattern of patchy, often multilobar, infiltrates which may exhibit cavitary change. In the USA, approximately $2 \%$ of patients admitted to the hospital for treatment of community-acquired pneumonia demonstrate microbiologic evidence of $S$. aureus 
infection (1). There is a slight predominance of methicillin sensitive species (MSSA) compared to methicillin resistant species (MRSA). Morbidity and mortality are both high, with over $80 \%$ of patients requiring care in the ICU, and a fatality rate of $13 \%$ (2).

Among patients admitted to the intensive care unit with a primary diagnosis of influenza, there is a $15 \%$ incidence of $S$. aureus pneumonia. Risk factors for co-infection in this setting include obesity, HIV infection, and immunosuppressive medication. There is a robust association between bacteremia and mortality (3). Early empiric antibiotic therapy with an agent active against $S$. aureus should be strongly considered for patients admitted to the ICU with influenza complicated by pneumonia, pending the return of blood and respiratory cultures.

${ }^{1}$ Charles VanHook, ${ }^{2}$ Kristin Dahlem, and ${ }^{1}$ Angela Taylor

${ }^{1}$ Longmont United Hospital, Longmont, Colorado USA

${ }^{2}$ Massachusetts College of Pharmacy and Health Sciences, Boston, Massachusetts USA

\section{References}

1. Jain $\mathrm{S}$, Self $\mathrm{WH}$, Wunderink $\mathrm{R}$, et al. Community-acquired pneumonia requiring hospitalization among U.S. adults. N Engl J Med. 2015 Jul 30;373(5):415-27. [CrossRef] [PubMed]

2. Self WH, Wunderink RG, Williams DJ, et al. Staphylococcus aureus communityacquired pneumonia: prevalence, clinical characteristics, and outcomes. Clin Infect Dis. 2016 Aug 1;63(3):300-9. [CrossRef] [PubMed]

3. Martin-Loeches I, J Schultz M, et al. Increased incidence of co-infection in critically ill patients with influenza. Intensive Care Med. 2017 Jan;43(1):48-58. [CrossRef] [PubMed] 